\title{
Enfoques psicológicos de la textura en la musicología norteamericanana
}

Pablo Fessel* CONICET/Universidad de Buenos Aires

\section{Resumo:}

El trabajo examina enfoques sobre la textura producidos en el campo de la musicología norteamericana desde la década del 40, los cuales establecen una articulación entre consideraciones psicológicas y teóricas. En particular se revisan los enfoques de Meyer y Tenney, la caracterización de la melodía de Rahn, el enfoque del análisis de escena de Bregman, y el modelo de análisis de Ravenscroft. El trabajo expone un capítulo del desarrollo de la conceptualización de la textura en el pensamiento musicológico contemporâneo.

Palavras-chave: textura musical; enfoques psicológicos; musicología norteamericana

* Pablo Fessel es Doutor en Artes por la Universidad de Buenos Aires (UBA). Se desempeña como investigador en el Consejo Nacional de Investigaciones Científicas y Técnicas (Argentina) y como docente en la UBA. Es director editorial de la Revista del Instituto Superior de Música de la Universidad Nacional del Litoral. Ha sido profesor de musicología en la UNL, y editor invitado del número 8 de la Revista Argentina de Musicología, publicada por la Asociación Argentina de Musicología. Publicó la compilación Nuevas poéticas en la música contemporánea argentina - Escritos de compositores. Buenos Aires, Biblioteca Nacional, 2007. 


\section{§1 Introducción}

El concepto de textura, una expresión del pensamiento musical del siglo XX, se muestra particularmente elusivo a la teorización, en una vacilación entre enfoques que lo entienden como un concepto relativo a la constitución formal de la simultaneidad musical y otros que lo relacionan con aspectos ligados a la sonoridad. En lugar de negar su opacidad, y exigirla con un esfuerzo constructivo, es conveniente abordar el concepto tomando como punto de partida las dificultades que presenta. Resulta esclarecedor, en tal sentido, acudir a la historia del concepto, esto es, reconstruir el desarrollo de su conceptualización en el pensamiento musical contemporáneo, en tanto allí se despliegan muchos de los problemas que el concepto de textura pone en juego. La remisión a la historia puede dilucidar también en qué medida la reflexión metamusical registra, directa o indirectamente, el impacto de desarrollos compositivos que le son contemporáneos.

Es verosímil suponer una vinculación entre la crisis del lenguaje musical en la primera mitad del siglo XX y el acento, en la música de concierto posterior a esa época, aplicado sobre materiales y procesos eminentemente 'auditivos'. Como si se hubiera producido un desplazamiento histórico de poéticas compositivas orientadas a la innovación de la sintaxis musical, a otras dirigidas a la exploración de fenómenos y procesos relacionados con la percepción. Esto podría explicar la consideración creciente, por parte de la musicología, de conceptos como el de textura, mediante categorías provistas por la psicología de la percepción.

El presente trabajo expone un capítulo del desarrollo histórico de la conceptualización sobre la textura en el pensamiento musicológico contemporáneo. Este capítulo incluye una variedad de enfoques elaborados en la musicología norteamericana a partir de la década del 40 , que se caracterizan 
por apoyar sus formulaciones analíticas en supuestos derivados de una psicología de la percepción auditiva. ${ }^{1}$

\section{§2 Antecedentes}

La primera consideración teórica del concepto de textura se produjo en el ámbito de la crítica musical inglesa, a comienzos del siglo XX, por parte de autores como C. Hubert Parry (1911), George Dyson (1923) y Donald Tovey (1941). Hasta entonces el término 'texture' había sido empleado en la crítica inglesa y angloparlante en general simplemente como una categoría de escritura, en expresiones como 'textura polifónica' o 'textura homofónica' ${ }^{2}$ El término carece de una entrada propia en la primera edición del diccionario editado por George Grove (1900), y se lo emplea sólo como traducción del vocablo italiano 'tessitura' en su sentido corriente, a falta, según Henry Collins Deacon, el autor de la entrada correspondiente, de un equivalente directo en inglés.

La inclusión de una entrada dedicada al concepto en el Harvard Dictionary of Music, escrita por Willi Apel (1944) cuenta como una institucionalización del término en la musicología angloparlante. ${ }^{3}$ La textura resulta para Apel de la interacción de dos elementos, un elemento horizontal (la melodía) y un elemento vertical (la armonía). Estos elementos configuran un espacio delimitado por dos categorías extremas, la polifonía estricta y la homofonía estricta, entre las cuales se ubica una "gran variedad de texturas intermedias", tales como diversas texturas pianísticas del siglo XIX así como lo

\footnotetext{
${ }^{1}$ La fragmentación institucional de los estudios musicológicos en el ámbito norteamericano podría suscitar un interrogante acerca de la adscripción de los enfoques considerados en el trabajo al campo de la 'musicología'. Aludo a este campo en el sentido inclusivo con que se lo entiende en el medio académico hispanoamericano.

${ }^{2}$ Cf. por ejemplo Parry (1893, p. 193, 295) y Goetschius (1902, p. 82). Estas expresiones representan un equivalente de las 'categorías estilísticas' de Adler (1911). Sobre el origen del concepto de textura en la crítica musical inglesa, véase Fessel (2007).

${ }^{3}$ Una marca de esta institucionalización está dada por la incorporación del término a la caracterización del 'lenguaje musical', con atributos similares a los que recibe en Apel, en trabajos de orientación pedagógica como los de Liepmann (1953), Machlis (1955), Boyden (1956), Cooper (1957), Smith Brindle (1966) y Deri (1968).
} 
que Apel denomina texturas pseudo-contrapuntísticas del siglo XVI. Apel indica asimismo relaciones diagonales en la textura, ejemplificadas por fenómenos como la imitación contrapuntística y la anticipación, entendida como ornamento melódico. El enfoque de Apel es heredero de las taxonomías elaboradas por la musicología comparada alemana, llevadas a cabo a efectos de precisar la naturaleza y alcances de la multilinealidad (Mehrstimmigkeit). ${ }^{4}$

En las décadas posteriores a la segunda guerra mundial, con la constitución en Estados Unidos de la Music Theory como un ámbito relativamente autónomo de pensamiento musical, la textura será objeto de una teorización análoga a la de otras dimensiones del análisis, tales como la armonía, el ritmo o la forma. ${ }^{5}$

\section{§3 Aproximaciones psicológicas de la textura}

La psicología de la música norteamericana se caracteriza por una orientación fundamentalmente empirista. Los enfoques psicológicos se constituyen a partir de una restricción al ámbito de la percepción, en una identificación que va a permitir el paso de la psicología conductista, dominante 10 en los años 50 en EE.UU., a los enfoques cognitivos preponderantes desde fines de la década del 70. El concepto de textura va a ser objeto en estos enfoques de una asociación con los principios perceptivos derivados de la Gestalttheorie alemana.

\section{§3.1 Textura como figura y fondo}

Leonard Meyer dedica unas páginas a la textura musical en su influyente libro sobre la semanticidad musical (1956). No hay allí una teoría desarrollada de la textura, sino que ésta resulta incluida en una teoría general de la audición

\footnotetext{
${ }^{4}$ La contraposición entre la horizontalidad y la verticalidad deriva en última instancia de una contraposición análoga establecida desde el siglo XVIII entre el contrapunto y la armonía (Eggebrecht, 1979. p. 313-314, v. 3).

${ }^{5}$ Cf. por ejemplo LaRue (1970), Berry (1987), Cogan y Escot (1976) y Delone (1975).
} 
musical. La textura está concebida en términos de una estructura representacional, esto es, una estructura entendida como derivada de una imposición de organizaciones sobre los materiales sensoriales. Constituye en este sentido el primer estudio de la textura desde lo que se va a constituir décadas más tarde como una tradición cognitiva. Esta nueva perspectiva teórica, que supone en líneas generales un desplazamiento de un punto de vista positivista como el de Apel a uno empirista, implica en particular un desplazamiento relativo al estatuto mismo de la textura musical. Ésta pasa de constituirse como un concepto ligado a la escritura musical a hacerlo como un concepto propio de la escucha.

Las categorías que van a dar cuenta de la asignación perceptiva están tomadas de la teoría de la Gestalt. Meyer reemplaza la dicotomía entre las dimensiones horizontal y vertical de Apel por otra dicotomía constituida por la oposición figura-fondo. ${ }^{6}$ La textura se define como "los modos en los cuales la mente agrupa estímulos concurrentes en figuras simultáneas, una figura con acompañamiento (fondo) etc." (Meyer, 1956, p. 185)..$^{7}$ En consecuencia con esta definición, de tipo extensional, Meyer elabora una taxonomía que contiene categorías como: (1) una figura sin fondo; (2) varias figuras sin fondo (polifonía); (3) una o más de una figura acompañada por un fondo (textura homofónica); (4) fondo solo; (5) superposición de motivos con poca independencia real de movimiento (la heterofonía). Combinaciones entre estas categorías, señala Meyer, son asimismo posibles. Las categorías 1 a 4 se basan en dos principios clasificatorios: el primero (la oposición figura-fondo, en todas sus combinaciones) distingue las categorías 1, 3 y 4; el segundo (el número de figuras: una-más de una) distingue las categorías 1 y 2 . La categoría 5, por otra parte, se funda en un principio clasificatorio enteramente nuevo.

De modo análogo a la estructura melódica, objeto privilegiado de los principios perceptivos postulados, la textura establece relaciones de 'expectación'

\footnotetext{
${ }^{6}$ Este enfoque tiene antecedentes en estudios de psicoacústica como los de Wever y Truman (1928) y Vernon (1934).

${ }^{7}$ Las traducciones son nuestras.
} 
(entendidas como expectativas de ocurrencias musicales determinadas en mayor o menor medida en el decurso posterior del proceso musical) a partir de lo que Meyer caracteriza como situaciones de 'incompletitud' textural. Los principios perceptivos que rigen esas relaciones son dos, la continuación y el completamiento, vale decir, cierta forma de expectativa ya sea de continuidad o de clausura. Las situaciones de 'incompletitud' textural que ponen en juego tales principios son de tres clases. En primer lugar la expectativa de una recurrencia posterior de una textura presentada anteriormente, lo que constituye una expectativa formal más que textural en sentido propio. En segundo lugar, Meyer identifica texturas caracterizadas por un espaciamiento relativamente pronunciado entre sus componentes como generadoras de una expectativa de completamiento vertical. Por último, puede producirse la expectativa de ocurrencia de una 'figura', cuando la uniformidad del 'estímulo' musical conduce a que éste sea entendido como un puro acompañamiento.

El enfoque de Meyer representa uno de los primeros intentos de encuadrar el estudio del problema de la textura en un contexto teóricamente articulado. No obstante ello, admite al menos dos objeciones. En primer lugar, la transposición de principios perceptivos generales al campo de la audición estética supone en el fondo una negación de toda especificidad de la última respecto de la escucha cotidiana. ${ }^{8}$ En segundo lugar, el carácter universal, ahistórico, de los principios psicológicos postulados resulta sugestivamente asociado a formulaciones clasicistas.

\section{§3.2 El enfoque gestáltico de James Tenney}

Otra aproximación gestáltica es la de James Tenney, formulada años después (1988). Tenney se propone la elaboración de un nuevo sistema conceptual para la descripción y análisis musical de la música del siglo XX.

\footnotetext{
${ }^{8}$ De hecho, es dable suponer que tanto la condición no funcional de la audición estética, como su aplicación hacia un objeto históricamente determinado, conllevaría alguna especificidad de sus procesos respecto de los de la escucha cotidiana.
} 
El trabajo de Tenney se ordena en tres secciones. La primera, denominada "Los nuevos materiales", gira alrededor de dos preguntas. La primera surge a partir del reconocimiento de una diferencia en la complejidad de los materiales sonoros que funcionan como elementos básicos de la composición. Tenney adopta para todos esos elementos la designación común de clang, y formula una pregunta por su constitución en tanto que unidad compositiva elemental. La segunda es una pregunta por la continuidad en música. Estas preguntas se formulan una vez que categorías como nota y motivo han perdido relevancia como designación de las unidades elementales de la composición y la continuidad musical no descansa ya en principios vinculados a la elaboración temática. Las preguntas de Tenney son, en rigor, una y la misma: “¿cómo se constituyen unidades musicales?", tal como este interrogante se plantea en los ámbitos del instante musical y del despliegue temporal de la obra. En otros términos, la pregunta podría parafrasearse del siguiente modo: “¿cómo se distingue una unidad de otras unidades sonando simultánea y sucesivamente?"

La respuesta a estos interrogantes tiene una dirección confluyente: se orienta al ámbito de la percepción. La característica más general común a todos los tipos de clang es el hecho de ser percibidos como unidades. A partir de este desplazamiento, Tenney introduce tres nociones. La primera ocupa el lugar de conceptos tales como las de 'sonido', 'configuración sonora' o 'idea musical' (en una referencia expresa al concepto de Schönberg). Se trata de una noción de clang entendido como cualquier sonido o configuración sonora "percibida como unidad musical primaria" (Tenney 1988, p. 23). Las partes componentes de un clang tales como notas, acordes o sonidos de cualquier naturaleza se denominan elementos. Por último una sucesión de 'clangs' con algún grado de unidad y singularidad que la haga constituirse en un 'gestalt musical' en un nivel perceptivo o temporal mayor se denomina secuencia.

La segunda sección está dedicada al análisis de los factores de cohesión y separación de unidades. Se trata, en líneas generales, de las leyes de la Gestalt transpuestas al plano de la percepción musical. Tenney procede a una distinción entre factores sin implicancias formales, a los que caracteriza como estadísticos - 
objeto de la segunda sección -, y factores con implicancias formales, a los cuales caracteriza como morfológicos - objeto de la tercera.

Los primeros son objeto de variación en magnitudes ligeras. El primer factor considerado es el de proximidad, que Tenney considera en el orden temporal. Este principio establece que "en una serie de elementos sonoros, aquellos simultáneos o contiguos tenderán a formar unidades, mientras que la relativa separación en el tiempo producirá segregaciones." (p. 29). El segundo factor es el de similitud, que Tenney aplica a los órdenes del timbre y de la altura. La similitud tímbrica o de altura opera cohesivamente, mientras que la correlativa disimilitud lo hace en forma disociativa.

A estos factores se agregan factores secundarios tales como el de intensidad (entendida como alguna forma de prominencia en una dimensión musical: un ascenso en la altura, un incremento en el nivel dinámico, en el tempo etc.), la repetición, así como factores a los cuales Tenney denomina el 'conjunto objetivo' y el 'conjunto subjetivo'. El primero comprende las expectaciones o anticipaciones surgidas en una experiencia musical a partir de eventos anteriores en la misma obra. El segundo toma en consideración aquellas derivadas de la experiencia musical previa del oyente.

Tales factores operan en cada configuración musical en forma simultánea, aunque no siempre con la misma importancia. Su acción en la organización perceptiva varía entre una completa congruencia o refuerzo mutuo, un efecto ambiguo, y la constitución de configuraciones ambivalentes, producidas por relaciones antitéticas entre aquellos (p. 32).

La tercera sección está dedicada a los factores morfológicos. Tenney se ocupa aquí de las relaciones de isomorfismo o heteromorfismo entre los materiales, así como del papel cohesivo de los factores. Procede asimismo a una reformulación de las categorías estilísticas tradicionales (categorías tales como monofonía, polifonía) de acuerdo con los principios analíticos introducidos anteriormente.

Si en el enfoque de Meyer el desplazamiento al punto de vista de la percepción musical suponía una sustitución de las categorías estilísticas por otras 
como las de figura y fondo (en rigor, la definición de las primeras bajo los términos de las segundas), en el de Tenney se procede a la suspensión de toda instancia categorial. ${ }^{9}$ Tenney aborda el problema de la constitución de la simultaneidad y de la sucesión musical a partir de las propiedades paramétricas 'puras' de los materiales. Ese elemento de nominalismo representa el momento crítico del enfoque. Si bien esos materiales no están todavía considerados en su carácter histórico (su naturaleza se identifica con propiedades más próximas a la acústica que a la historia), el enfoque de Tenney, al desembarazarse de los presupuestos de los enfoques categoriales, presenta el problema de la conformación de la textura con un grado inédito de profundidad. ${ }^{10}$ Esa 'suspensión' de las categorías estilísticas tradicionales, tanto como de las de figura y fondo, con las cuales Meyer había intentado reemplazarlas, análoga

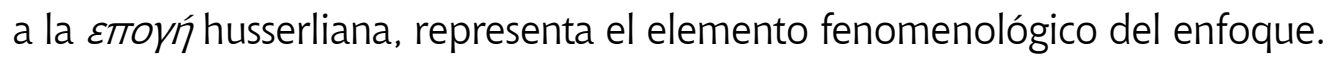

\section{§3.3 La constitución de la melodía}

Jay Rahn adopta un punto de vista similar al de Tenney, en un artículo titulado “¿Dónde está la melodía?” (1982). Los paralelismos entre un enfoque y otro son varios. Rahn comienza su trabajo, parafraseando el título, con la siguiente formulación:

[D]ado un ordenamiento potencialmente confuso de sonidos simultáneos presentados en una ejecución o en una partitura, ¿cómo sabemos exactamente qué sucesiones de notas merecen recibir el estatuto de unidades melódicas? (p. 3).

El momento escéptico implícito en la pregunta por la constitución de la melodía en cuanto tal reitera, en un nivel más alto de organización, el de Tenney,

\footnotetext{
${ }^{9}$ Aunque en la tercera sección Tenney se ocupa brevemente de las categorías, éstas no desempeñan un papel regulativo. La reformulación de las categorías en términos de los factores propuestos cumple la función de saldar cuentas con la tradición.

${ }^{10}$ La reducción de los materiales musicales, cargados de estilo e historia, a la condición de una pura materia, permitiría establecer un vínculo entre este marco conceptual y la estética de las corrientes experimentales norteamericanas. La situación de Tenney como compositor postcageano apoyaría esta relación. (Debo a Omar Corrado la sugerencia de esta relación).
} 
referido a la constitución de las unidades texturales y formales de las obras musicales contemporáneas. La pregunta de Rahn constituye simplemente un paso heurístico orientado a la explicitación de los criterios a partir de los cuales se construye perceptivamente una melodía. Para ello Rahn establece una distinción entre las categorías de 'parte' y de 'melodía'. La primera está definida como "una sucesión de notas próximas o similares entre sí (y distantes o diferentes de otras) en algunos aspectos" (p. 3).

La melodía está concebida por su lado como una parte relativamente compleja o prominente. Consecuentemente con la posición nominalista, la distinción entre partes y melodías no constituye una distinción categorial sino una de grado. Rahn rechaza la posibilidad de una distinción basada en criterios cuantificables, en tanto las variables que determinan la constitución de estas categorías son de naturaleza "cualitativa o comparativa, esto es, dos valores pueden ser iguales o diferentes o uno puede ser mayor o menor que otro en algún sentido, pero no hay un modo razonable de asignar un valor numérico para expresar aquellas similitudes o diferencias." (p. 4). Esa imposibilidad permite establecer una diferencia de naturaleza entre unidades texturales (tales como partes o melodías) y unidades sintácticas (tales como intervalos o acordes), susceptibles de cuantificación.

Rahn pasa a identificar las variables que determinan respectivamente la constitución de una parte y la de una melodía. Entre las primeras señala la identidad de timbre, la relativa proximidad de altura, el aislamiento relativo de las alturas en la disposición vertical de sonidos, la relativa proximidad de ataque y la similitud de diseño, entendido como variable que toma en cuenta los aspectos vinculados a la articulación. Entre las variables que determinan la constitución de una melodía Rahn indica la variedad en el contenido y contorno de altura, la intensidad así como la ubicación en el extremo registral.

Rahn inscribe su enfoque en la tradición gestáltica de la teoría de la música y establece una serie de paralelismos entre las unidades musicales analizadas y unidades del ámbito de la percepción visual. Las partes corresponden a grupos perceptivos cuyo agrupamiento se alcanza mediante la percepción de elementos de similitud (de timbre, de posición textural, de diseño) 
y de proximidad (de alturas simultáneas y sucesivas y de ataques). Respecto de la melodía, la intensidad representa un equivalente del brillo, así como la complejidad se corresponde con la densidad textural en las imágenes. La melodía se constituye así como figura, cuyos extremos pueden considerarse como los límites que definen los contornos de las figuras en la percepción visual. En síntesis, las partes constituyen el correlato de los agrupamientos de la percepción visual, y las melodías, un análogo de las figuras.

El aporte más significativo del trabajo de Rahn está dado por la pregunta por los criterios de la constitución de la melodía en cuanto tal, en la medida en que se trata de una pregunta ajena a la Melodielehre tradicional. Ésta se ocupaba de la morfología de la melodía, pero daba por supuesta su constitución misma en cuanto tal dentro de la simultaneidad musical. ${ }^{11}$

\section{§3.4 El análisis de la escena auditiva}

Las intuiciones que en Tenney derivan de un punto de vista fenomenológico, y que suponen un carácter especulativo considerable, surgen en el estudio de Albert Bregman (1990) como resultado de una metodología experimental. Este trabajo, síntesis de investigaciones realizadas en el campo de la psicoacústica desde los años 70 , está centrado en el análisis de lo que Bregman denomina la escena auditiva general. Las consideraciones vinculadas a la textura tienen lugar en tanto la simultaneidad musical se constituye como una escena auditiva de un tipo particular. De hecho, Bregman deriva expresamente la constitución de diferentes organizaciones musicales, entre ellas la de las estructuras texturales, de principios generales, esto es, no específicamente musicales, de organización auditiva. Tales principios subyacentes, dice Bregman, "trascienden a cualquier estilo musical. Si esto es cierto" - agrega - "los diferentes estilos musicales simplemente dan a los principios subyacentes la oportunidad de operar en mayor o menor grado, pero no pueden modificarlos." (p. 457).

11 Los trabajos de Meyer (1973) y de Narmour (1990), que aspiran a refundar la Melodielehre bajo principios cognitivos, presentan la misma insuficiencia. 
Esta hipótesis fuerte - la homología entre los órdenes de la percepción auditiva general y la percepción musical, si no la determinación de la segunda por la primera - hace pertinente una revisión detallada del enfoque.

\section{\$3.4.1 La escena auditiva}

El concepto de escena auditiva, punto de inicio y a la vez objeto de la teoría, se define como la representación mental de los acontecimientos ocurridos en torno del sujeto, en tanto éstos se manifiestan acústicamente. La noción de acontecimiento ocupa de este modo el lugar que corresponde, en el orden de la percepción visual, al concepto ontológico de objeto entendido como unidad de la escena visual. Mientras que el análisis de la escena visual se orienta a la descripción de objetos (entendidos ahora como unidades de la percepción visual), el análisis de la escena auditiva lo hace hacia la descripción de la representación sensible (auditiva) de los acontecimientos. Sin embargo, la unidad de tales representaciones no se caracteriza como 'sonido' sino como 'corriente auditiva' (auditory stream).

¿Por qué no denominar [a la unidad perceptiva que representa un único acontecimiento] 'sonido'? [...] Nuestras representaciones mentales de eventos acústicos pueden ser hasta tal punto múltiples como no lo sugiere el término 'sonido'. Al acuñar un término nuevo, 'corriente', somos libres de cargarlo con las propiedades teóricas que resulten apropiadas. (Bregman, p. 10).

La sustitución terminológica expresa así de modo inequívoco el aspecto anticategorial del enfoque.

El análisis de la escena auditiva incorpora, en líneas generales, los principios formulados por la teoría de la Gestalt, reinterpretados como reglas funcionales. Mientras que esta última concibe los principios como parte de un sistema autosuficiente cuyo objeto es la organización del estímulo, aquélla parte de premisas ecológicas: el análisis relaciona el proceso de organización de la evidencia auditiva con el problema que el 
entorno plantea al sujeto en tanto éste trata de construir descripciones eficaces de situaciones ambientales.

Como en la Gestalttheorie, el análisis de la escena auditiva guarda un cierto número de correspondencias con el de la escena visual. Sin embargo, la especificidad de la primera respecto de la segunda radica en que las unidades de la escena auditiva son 'transparentes':

Los sonidos son transparentes porque cuando dos de ellos se hallan activos al mismo tiempo, sus propiedades se superponen. En la visión, en el caso de objetos opacos, si un objeto más próximo cubre a otro, sólo se presentan al ojo las propiedades del más próximo [...] (Bregman, p. 382).

A diferencia de lo que ocurre en el caso de la visión, los diferentes objetos auditivos suman su energía en la señal auditiva.

\section{\$3.4.2 La constitución de las corrientes auditivas}

Las corrientes auditivas, como unidades del análisis de la escena auditiva, se constituyen a partir de dos tipos de proceso, a los cuales Bregman denomina integración y disgregación (segregation). Cada uno de ellos se aplica por su parte a dos órdenes, el orden de la simultaneidad y el de la sucesividad. De ello resultan ámbitos específicos del análisis de escena: la formación de unidades, la integración secuencial, la integración de la simultaneidad (denominada en ocasiones integración espectral), y la disgregación de corrientes auditivas (auditory stream segregation). ${ }^{12}$

La construcción de una determinada representación de la escena auditiva pone en juego dos operaciones: en primer lugar, un proceso de partición del conjunto de componentes concurrentes en distintos subconjuntos, que los ubica en diferentes corrientes, y, en segundo lugar, un proceso de agrupamiento secuencial, que dispone algunos de los componentes del espectro que siguen unos a otros en el tiempo dentro

12 Bregman identifica por su parte dos clases de restricciones sobre la formación de representaciones perceptivas: restricciones primitivas y restricciones más sofisticadas denominadas esquemas (1990, 38 ss.). Esta distinción intenta dar cuenta de variantes debidas a principios de acción presumiblemente innatos, en el primer caso, y a principios derivados de la experiencia, en el segundo. 
de la misma corriente perceptiva. En otros términos, el proceso de análisis de escena descompone el conjunto de energía entrante en un gran número de análisis separados, correspondientes a momentos particulares y regiones de frecuencias particulares del espectro. Posteriormente, el sistema auditivo procede a una operación de integración que asegura que cada agrupamiento derive del mismo evento ambiental. Esa integración, o agrupamiento, se lleva a cabo en dos dimensiones: a través del tiempo (en el caso de la integración secuencial) y a través del espectro (en el caso de la integración de la simultaneidad).

Cada uno de estos procesos cuenta con principios de organización específicos. Los factores determinantes para la constitución de las corrientes son la relativa separación de las frecuencias de los elementos sucesivos en el tiempo y su relativa proximidad temporal (la velocidad de la frecuencia). Esta primera observación establece dos caracteres esenciales de los factores implicados en el análisis de la escena auditiva. En primer lugar, los factores son relativos al conjunto de la evidencia. La representación precisa de lo que cuente como proximidad de altura dependerá de la extensión total de alturas presentes. En segundo lugar, los factores operan en forma competitiva: la proximidad temporal integra en un sentido, y la proximidad de frecuencias en otro. El resultado de ambos factores no necesariamente confluye. De ello resulta una diferencia entre agrupamientos relativamente fuertes - resultado de una acción confluyente de los factores - o relativamente débil - resultado de una acción no confluyente de los mismos factores. En tercer lugar, los factores operan, en ocasiones, en forma contradictoria. La tolerancia hacia la no-proximidad de frecuencias es tanto menor cuanto mayor es la velocidad de la secuencia. $Y$ alternativamente, a menor velocidad mayor es la posibilidad de integración de frecuencias alejadas. Refiriéndose ahora expresamente a la música, dice Bregman: "Tan pronto como se incrementa la separación entre las frecuencias, la secuencia debe ser ralentada para que el oyente sea capaz de experimentar todas las notas como parte de una corriente de sonido única y coherente." (p. 643).

La similitud tímbrica opera asociativamente, del mismo modo que la presunta procedencia espacial y la trayectoria (entendida como cambio 
gradual de cualquier naturaleza). Sin desempeñar un papel determinante, diferencias de intensidad pueden asumir una función delimitadora dentro de las señales.

Los factores que promueven el agrupamiento de las entradas secuenciales son rasgos que definen la similitud y la continuidad de sonidos sucesivos. Estos incluyen la frecuencia fundamental, su proximidad temporal, el diseño de su espectro, su intensidad, y su origen espacial aparente. Estos factores conforman una jerarquía, con niveles de incidencia diferentes sobre la integración de las secuencias. Escribe Bregman:

La coherencia de una secuencia puede ser interpretada en términos funcionales como una heurística del sistema auditivo. Esta heurística es equivalente a una apuesta [en el sentido] de que toda secuencia que exhiba continuidad acústica proviene probablemente de un único evento ambiental. (p. 649).

La interpretación funcional da cuenta así del sustrato explicativo ecológico del enfoque: el sistema opera eficazmente en la medida en que las unidades constituidas, resultado de ese proceso de disgregación e integración, se corresponden con acontecimientos del entorno. En otras palabras, el funcionamiento de la percepción auditiva se orienta a una correlación verdadera entre las propiedades de la representación y los acontecimientos ocurridos en el entorno ambiental.

Bregman identifica tres factores que producen una integración de la simultaneidad. El principio de armonicidad, en primer lugar, integra verticalmente los segmentos del espectro que pueden ser percibidos como armónicos de una misma fundamental. El principio gestáltico de 'destino común' da cuenta de la integración de elementos que son objeto de transformaciones en un sentido confluyente. El principio de correspondencia espacial, por último, integra elementos que provienen de la misma posición en el espacio. 


\section{\$3.4.3 La organización auditiva en la música}

En consecuencia con los principios formulados, Bregman se propone ilustrar fenómenos musicales particulares que ponen en evidencia la acción de los principios del análisis de escena:

Tanto las organizaciones secuenciales como simultáneas crean realmente ciertos aspectos de la experiencia musical. El agrupamiento secuencial crea ritmos y diferentes aspectos de la forma melódica. La organización vertical nos da no sólo la experiencia de los acordes sino también otras cualidades emergentes de los sonidos simultáneos, tales como el timbre, la consonancia y la disonancia. Esos fenómenos surgen cuando ciertos componentes acústicos se agrupan perceptivamente en la misma organización vertical. (p. 459).

La preponderancia del grado conjunto en la conformación de las líneas melódicas en la polifonía renacentista es interpretada por Bregman como derivada del efecto desintegrador de la separación de frecuencias. La 'polifonía implícita' de Bach, por su parte, supone el aprovechamiento de la acción contradictoria de los principios de proximidad temporal y de altura. ${ }^{13}$ La percepción de los acordes resulta tanto de las propiedades integradoras de la consonancia, como de la acción igualmente integradora en lo vertical de la

22 sincronía de ataques. La distintividad de las voces en el contrapunto deriva por su parte de la elusión de la sincronía rítmica y del cruce de las partes, tanto como de la direccionalidad convergente o la similitud tímbrica. Aspectos más elementales de la organización musical tales como la constitución de las notas mismas o la conformación del timbre resultan de un único proceso de partición de componentes simultáneos, responsable asimismo de la formación de corrientes.

\section{\$3.4.4 La textura como escena auditiva}

El punto de vista perceptivo instaura de este modo una correspondencia necesaria entre la organización de la textura musical y la de la escena auditiva. Ese posicionamiento por parte de Bregman presenta un elemento crítico, dado por lo que

\footnotetext{
${ }^{13}$ El problema está tratado en forma más extendida en Wright y Bregman (1987).
} 
se caracterizó anteriormente como el momento escéptico: se trata de una suspensión provisional en el análisis de la operación de toda categoría dada previamente. En otras palabras, el objeto se concibe por un instante como desprovisto de forma alguna. ${ }^{14}$ Pero ese momento crítico no se limita al plano del sujeto de conocimiento, sino que se extiende al objeto en sí mismo, el cual queda carente de determinaciones inherentes. Ese constituye precisamente el punto débil del enfoque: la constitución del material musical como objeto estético e histórico, por compleja que resulte la conjunción de tales determinaciones, resulta negada. Además, consecuentemente, es ignorado el carácter histórico del sujeto de la percepción. Se desconoce así la naturaleza histórica del material musical, tanto como su distancia irreductible respecto del hecho acústico.

\section{§4 Modelos de análisis textural}

Desde fines de la década del 60, los enfoques sobre la textura producidos en Estados Unidos se caracterizan por la formulación de modelos teóricos para su análisis. Uno de estos modelos, elaborado por Wallace Berry (1987), tuvo una significativa influencia en la teoría de la música norteamericana. ${ }^{15}$ El emplazamiento por parte de Berry de la textura como uno de los aspectos funcionales de la música, junto con el ritmo y la tonalidad, supuso una jerarquización de la primera sin precedentes en enfoques similares. Este modelo tuvo uno de sus desarrollos inmediatos en la tesis doctoral de Anne Hall, presentada en la Universidad de Michigan en $1971 .{ }^{16}$ Los estudios de Berry y Hall representan un paso hacia una concepción no tipológica de la textura. Las observaciones sobre los aspectos funcionales de la textura - los procesos de los cuales es objeto - no se orientan hacia la identificación de las funciones de los elementos constituyentes (melodía,

\footnotetext{
${ }^{14}$ Bregman da un paso más que Tenney y que Rahn, en la medida en que identifica por un momento audición musical con audición a secas.

${ }^{15}$ El trabajo de Berry, publicado en 1976, es, sin embargo, considerablemente anterior. El trabajo de Anne Hall (1971), se reconoce inspirado en un seminario sobre la textura dictado por Berry en la Universidad de Michigan.

${ }^{16}$ Los modelos de Berry y Hall están discutidos más ampliamente en Fessel (2006).
} 
acompañamiento etc.) en la textura sino hacia su papel en la forma musical. La primacía del concepto de línea supone por otra parte una diferenciación clara entre los conceptos de textura y de sonoridad. La textura se constituye así como un elemento de tipo formal en el orden de los materiales musicales.

Otro de estos modelos, de particular interés en el contexto de este trabajo, es el modelo de análisis textural propuesto por Brenda Ravenscroft (1993). Ravenscroft elabora una teoría de la textura orientada al análisis de $A$ mirror on which to dwell, de Elliott Carter, un ciclo de seis canciones para soprano y orquesta de cámara compuesto entre 1975 y 1976 y basado en poemas de Elizabeth Bishop. El trabajo de Ravenscroft es deudor de dos enfoques: los modelos texturales de Hall (1971) y Berry (1987), y la teoría del análisis de la escena auditiva desarrollada por Bregman (1990).

El concepto de corriente se convertirá en una pieza fundamental del enfoque. De hecho, se trata de un concepto empleado por Carter con relación a su propia música (Edwards, 1971, p. 101). Ravenscroft concluye, diferenciándose de Berry, en la necesidad de una teoría de la textura cuyos componentes últimos no sean necesariamente líneas instrumentales. En este aspecto se pone en evidencia el aporte más importante del enfoque de Bregman.

La teoría de Ravenscroft se despliega en tres grandes apartados. El primero se ocupa del concepto de corriente y de las características que lo definen. El segundo apartado se ocupa a las relaciones entre las corrientes. El tercero lo hace de las funciones, musicales y no musicales, que pueden asumir las unidades texturales.

Ravenscroft caracteriza una corriente como

una continuidad distintiva, compuesta de una serie de eventos que se perciben como una continuidad temporal porque son reconociblemente similares y porque se diferencian de eventos pertenecientes a otras posibles corrientes concurrentes. (p. 33).

Las corrientes se definen en función de la similitud entre los eventos participantes en cuanto a determinados aspectos musicales, similitud que los asocia entre sí, así como por la disimilitud en otros aspectos respecto de eventos 
en otras corrientes, por la cual se disocian de los últimos. El criterio para evaluar el grado de similitud y disimilitud es comparativo: no existe un parámetro intrínseco sino que los criterios de similitud son relativos a los de disimilitud (p. 32).

Hay tres aspectos de las corrientes que contribuyen a su percepción y distinción, aspectos que Ravenscroft denomina propiedades, comportamientos y procesos. Esos aspectos se ordenan en una jerarquía de inclusión.

Las propiedades son características que pueden ser percibidas y evaluadas a cada momento dado, o dentro de un intervalo de tiempo breve. Una propiedad define una corriente si permanece en forma relativamente constante a lo largo de un intervalo de tiempo relativamente largo, o, en otras palabras, si esta corriente puede ser percibida como la misma cuando se la considera en varios momentos a lo largo de un determinado intervalo de tiempo. Ravenscroft distingue entre propiedades primarias, capaces por sí mismas de constituir una corriente, y propiedades secundarias, que carecen de esa capacidad y simplemente refuerzan la acción asociativa de las primeras. Entre las propiedades primarias se incluyen el timbre, el registro y la altura. Entre las propiedades secundarias lo hacen la densidad registral, los ataques y la intensidad.

Los comportamientos suponen cambios consistentes en las propiedades de las corrientes y requieren una cierta duración para ser percibidos. Los cambios que dan lugar a un comportamiento no deben ser tan profundos, y al menos una de las propiedades primarias debe permanecer inmodificada, de modo que no se perciba una nueva corriente. Al igual que en el caso de las propiedades, Ravenscroft distingue entre comportamientos primarios y secundarios. El primero está ejemplificado con el pulso: cuando los ataques de los eventos conforman un pulso, este comportamiento rítmico es determinante en la constitución de una corriente. Los comportamientos secundarios incluyen fenómenos como la contigüidad entre dos eventos, ascensos y descensos en el contorno de una línea melódica, cambios en la densidad de ataques, incrementos o decrecimientos de densidad registral y cambios en la intensidad.

Los procesos, por último, suponen la percepción de cambios consistentes en los comportamientos de las corrientes a lo largo de un período temporal 
extenso (p. 33-34). Si los cambios fueran muy profundos, no se percibiría una única corriente continua sino la sustitución de una corriente por otra. A efectos de que se escuche un proceso dentro de una corriente, algunas de las propiedades y comportamientos deben permanecer constantes mientras las otras cambian ( $p$. 70-71). Uno de los ejemplos de proceso que da Ravenscroft es la intermitencia, esto es, la constitución de una corriente como una sucesión de gestos separados por silencios, donde la percepción de una sucesión como una corriente interrumpida depende de la continuidad dada por los otros aspectos que la definen como tal.

En síntesis, los comportamientos derivan de cambios en las propiedades y los procesos de cambios operados por su parte sobre los primeros.

Las relaciones entre corrientes que reconoce la teoría de Ravenscroft toman la forma de una combinación. La teoría identifica dos clases de combinación en la música de Carter: la alternancia, en la cual cada corriente procede intermitentemente, y la concurrencia, caracterizada por la simultaneidad entre dos (o más) corrientes. Las combinaciones de corrientes son, por su parte, objeto de comportamientos y de procesos. Entre los últimos se reconocen casos de fusión, división, foco, emulación, compensación, entre otros.

Tanto las corrientes como los comportamientos y los procesos asumen papeles funcionales. Ravenscroft distingue entre funciones simbólicas y funciones musicales. Entre las primeras analiza la representación por parte de las corrientes de personajes, y la de acciones por parte de los procesos. Las funciones musicales susceptibles de ser representadas por las unidades texturales son, por su parte, el clímax, la cadencia, la articulación estructural y la prolongación.

El aspecto más significativo del aporte de Ravenscroft está dado por el desplazamiento del concepto de línea, de la ubicación central con que contaba en las teorías corrientes de la textura, a un emplazamiento secundario. Ravenscroft, de acuerdo con Bregman, expone, en su análisis de las canciones de Carter, una concepción de acuerdo con la cual la línea representa una de las formas de constitución de las corrientes auditivas, entre otras formas 
posibles. ${ }^{17}$ Ello supone un paso más en el camino iniciado por Berry hacia una caracterización crítica de la linealidad, y representa en tal sentido un punto extremo en el desarrollo del nominalismo. "La teoría textural que voy a proponer no hace casi uso de terminología tradicional" anuncia Ravenscroft al comienzo de su trabajo (p. 12). Se trata de la utopía de una teoría sin categorías. Pero ese gesto no guarda consistencia con el conjunto del enfoque. La transformación, por parte de Ravenscroft, de las estipulaciones de Bregman sobre el carácter integrador o desintegrador de distintos factores musicales en una taxonomía rígida de propiedades supone el establecimiento de un nuevo sistema categorial. El nominalismo analítico se revela dirigido en oposición no a las categorías en cuanto tales, sino a su carácter histórico.

\section{$\S 5$ Consideraciones finales}

La elaboración de modelos teóricos de la textura implica la extensión hacia esta última de un tipo de enfoque corriente en otros dominios de la teoría de la música, tales como la armonía, el ritmo, o la forma. Las perspectivas psicológicas son, por su parte, particularmente renovadoras entre los enfoques sobre la textura producidos en el ámbito de la musicología norteamericana. Es probable que el desplazamiento del punto de vista, desde el posicionamiento tradicional de la teoría de la música, centrado en la partitura, hacia la audición, haya posibilitado una crítica de las nociones heredadas relativas a la textura, examinada ahora desde un punto de vista para el cual las categorías tradicionales se mostraban insuficientes, o sencillamente inadecuadas.

Pero ese mismo desplazamiento abre algunos interrogantes. Uno de ellos es la relativa especificidad de la audición musical (estética) respecto de la audición corriente, una distintividad que aconsejaría examinar cuidadosamente la transposición de categorías perceptivas de la última a la primera. En segundo lugar, se manifiesta una suerte de naturalización de la escucha, que desestima el papel que en ella desempeña la historia; esa negación de lo histórico en algunos

\footnotetext{
17 Una consideración análoga de la linealidad había sido desarrollada, en el ámbito de la musicología comparada, por Ficker (1930).
} 
enfoques, tanto del objeto como del sujeto de la percepción, redunda, sugestivamente, en formulaciones clasicistas. Por último, cabe preguntarse por la relevancia general de los principios perceptivos para la conceptualización de la textura, en la medida en que no se examine hasta qué punto se transfiere por esta vía una reflexión derivada de cierto tipo de composición, perceptivamente orientada, a un universo considerablemente diverso de músicas.

\section{Referencias}

ADLER, Guido. Der Stil in der Musik. Leipzig: Breitkopf \& Härtel, 1911. [Reimpresión de la 2da. ed. de 1929, Walluf, Sändig, 1973].

APEL, Willi. Texture. En: Harvard Dictionary of Music. Cambridge: Harvard University Press, 1944. p. 742-43. [2da. ed. ampliada, 1969, p. 842].

BERRY, Wallace. Texture. En: Structural Functions in Music. 2da. ed. New York: Dover, 1987. p. 184-300. [1ra. ed. 1976].

BOYDEN, David. An Introduction to Music. New York: Knopf, 1956.

BREGMAN, Albert. Auditory Scene Analysis. Cambridge, Mass.: MIT Press, 1990.

COGAN, Robert; ESCOT, Pozzi. Sonic Design. The Nature of Sound and Music. Englewood Cliffs: Prentice-Hall, 1976.

COOPER, Grosvenor. Learning to listen. Chicago: The University of Chicago Press, 1957.

DELONE, Richard. Timbre and texture in 20th-century music. En: DE LONE, R.; WITTLICH, G. E. (Eds.). Aspects of Twentieth-Century Music. Englewood Cliffs: Prentice-Hall, 1975. p. 66-207.

DERI, Otto. Exploring Twentieth-Century Music. New York: Holt, Rinehart \& Winston, 1968.

DYSON, George. The Texture of Modern Music. Music \& Letters, v. IV, n. 2, p. 107-118, 1923; n. 3, p. 203-218, 1923; y n. 4, p. 293-312, 1923.

EDWARDS, Allen. Flawed Words and Stubborn Sounds: A Conversation with Elliott Carter. New York: Norton, 1971.

EGGEBRECHT, Hans-Heinrich. Polyphonie. En: DAHLHAUS, C.; EGGEBRECHT, H. H. (Eds.). Brockhaus Riemann Musiklexikon. Wiesbaden: Brockhaus, 1979.

FESSEL, Pablo. El concepto de textura en la teoría de la música norteamericana. Revista Argentina de Musicología, n. 7, p. 117-145, 2006. . La doble génesis del concepto de textura. Revista Eletrônica de Musicologia, n. 11, 2007.

FICKER, Rudolf. Primäre Klangformen. Jahrbuch der Musikbibliothek Peters für 1929, v. 36. p. 21-34, 1930.

GOETSCHIUS, Percy. Counterpoint Applied in the Invention, Fugue, Cannon and Other Polyphonic Forms. New York: Schirmer, 1902. 
GROVE, George (Ed.). A Dictionary of Music and Musicians. London: Macmillan, 1900.

HALL, Anne. Texture in Violin Concertos of Stravinsky, Berg, Schoenberg, and Bartók. Tesis doctoral - University of Michigan, 1971.

LARUE, Jan. Sound. En: Guidelines for Style Analysis. New York: Norton, 1970. p. 23-31. [Ed. en español: El sonido. En: Análisis del estilo musical. Trad. de P. Purroy Chicot. Barcelona: Labor, 1989. p. 17-29].

LIEPMANN, Klaus. Language of music. New York: Ronald Press, 1953.

MACHLIS, Joseph. The Enjoyment of Music. An Introduction to Perceptive Listening. New York: Norton, 1955.

MEYER, Leonard B.. Explaining Music. Essays and Explorations. Berkeley: University of California Press, 1973.

. En: Emotion and Meaning in Music. Chicago: The University of Chicago Press, 1956. p. 185-96.

NARMOUR, Eugene. The Analysis and Cognition of Basic Melodic Structures. The ImplicationRealization Model. Chicago: The University of Chicago Press, 1990.

PARRY, C. Hubert. The Art of Music. London: Kegan Paul, 1893.

. Texture. En: Style in musical art. London: Macmillan, 1911. p. 173-206.

RAHN, Jay. Where is the melody?. In Theory Only, n. 6, p. 3-19, 1982.

RAVENSCROFT, Brenda. Texture in Elliott Carter's "A mirror on which to dwell". Tesis doctoral - University of British Columbia, 1993.

SMITH BRINDLE, Reginald. Serial Composition. London: Oxford University Press, 1966.

TENNEY, James. Meta + Hodos. A Phenomenology of 20th-Century Musical Materials and an Approach to the Study of Form. En: Meta + Hodos. A Phenomenology of 20thCentury Musical Materials and an Approach to the Study of Form. And META Meta + Hodos. 2da. ed. Oakland: Frog Peak Music, 1988. p. 1-97. [1ra. ed. 1964].

TOVEY, Donald. Musical Textures. En: A Musician Talks. London: Oxford University Press, 1941.

VERNON, P. E. Auditory Perception: I. The Gestalt Approach. British Journal of Psychology, n. 25, p. 123-139, 1934.

WEVER, Ernest G.; TRUMAN, Stanley. The Course of the Auditory Threshold in the Presence of a Tonal Background. Journal of Experimental Psychology, v. XI, n. 2, p. 98-112, 1928.

WRIGHT, James; BREGMAN, Albert. Auditory stream segregation and control of dissonance in polyphonic music. En: MCADAMS S. (Ed.). Music and Psychology: a Mutual Regard. London: Harwood, 1987. p. 63-92.

Pablo Fessel: pfessel@gmx.de

Recebido e aprovado em 05 de junho de 2008 